\title{
Editorial
}

\section{Advanced Mathematics and Numerical Modeling of IoT}

\author{
Young-Sik Jeong, ${ }^{1}$ Mohammad S. Obaidat, ${ }^{2}$ Jianhua $\mathrm{Ma}^{3}$ and Laurence T. Yang ${ }^{4}$ \\ ${ }^{1}$ Department of Multimedia Engineering, Dongguk University, Seoul 100-715, Republic of Korea \\ ${ }^{2}$ Department of Computer Science and Software Engineering, Monmouth University, West Long Branch, NJ 07764-1898, USA \\ ${ }^{3}$ Faculty of Computer and Information Sciences, Hosei University, Tokyo 102-8160, Japan \\ ${ }^{4}$ Department of Computer Science, St. Francis Xavier University, P.O. Box 5000, Antigonish, NS, Canada
}

Correspondence should be addressed to Young-Sik Jeong; ysjeong@dongguk.edu

Received 13 October 2014; Accepted 13 October 2014

Copyright (C) 2015 Young-Sik Jeong et al. This is an open access article distributed under the Creative Commons Attribution License, which permits unrestricted use, distribution, and reproduction in any medium, provided the original work is properly cited.

Recent advances in the Internet of Things (IoT) have posed great challenges to computer science and engineering. Internet of Things systems manage huge numbers of heterogeneous sensors and/or mobile devices which continuously mobile of the states of real-world objects, and most data are generated automatically through mobile networking environments. Internet of Things frameworks might help support the interaction between "things" and allow for more complex structures like Distributed/Grid/Cloud computing and the development of Distributed/Grid/Cloud applications. Currently, some Internet of Things frameworks seem to focus on real-time data logging solutions, which are offering some basis to work with many "things." Future developments might lead to specific software development environments to create the software to work with the hardware used in the Internet of Things [1-3]. IoT is also a novel paradigm that is rapidly gaining in the scenario of wireless sensor networks and wireless telecommunications. The basic idea of this concept is the pervasive presence around our life style of a variety of things or objects $[2,4,5]$.

The advanced mathematics and numerical modeling of IoT is being driven by and used in a wide range of academic, research, and commercial application areas. This use is producing important new practical experience in a variety of different problem domains in each of these areas. There are also new computational methods in these research fields. As an enabler, this technology is leading to a rapid growth in both scientific and information applications that will, in turn, enable additional requirements for the advanced mathematics and numerical modeling of Internet of Things to be identified. These will impact academia, business, and education $[1,3,5]$.

This special issue aims to provide advanced theory and application for researchers and practitioners to contribute original research and review articles that present the stateof-the-art research outcomes, practical results, latest findings, and future evolutions of mathematics in IoT applications.

We have received many manuscripts and the published manuscripts with high quality were finally selected for this special issue. Each manuscript selected was blindly reviewed by at least three reviewers consisting of guest editors and external reviewers. We present a brief overview of each manuscript in the following.

Recent advances in mathematics and numerical modeling of Internet of Things have created new topics of interest including the following: (1) mathematical and numerical modeling of smart city including smartphone real-world and mobile networks, (2) optimization methods, mathematics modeling for smart Grid with Cloud computing, (3) numerical analysis for security and emergencies in IoT, (4) methods for improving efficiency or accuracy of M2M applications and vehicle autodiagnosis, (5) adaptive and dynamic algorithms for home automation and e-health applications, (6) computational models of communication mechanisms for mobile networks in IoT, and (7) advanced modeling for IoT applications as mobile/vehicle ad hoc networks and mobile sensor networks in CPS (cyber physical system). 
In these several topics of the advanced mathematics and numerical modeling of IoT (Internet of Things), some articles proposed the following.

D. Lim et al. proposed a fall-detection algorithm using 3-axis acceleration. The fall-feature parameters, calculated from the 3-axis acceleration, are applied to a simple threshold method. Then, the falls that are determined from the simple threshold are applied to the HMM to distinguish between falls and ADLs. The results from a simple threshold, HMM, and the combination of the simple method and HMM are compared and analyzed.

H.-S. Ham et al. applied a linear support vector machine (SVM) to detect Android malware and compare the malware detection performance of SVM with that of other machine learning classifiers. Through experimental validation, they show that the SVM outperforms other machine learning classifiers.

J. Park and M.-J. Lee presented a context distribution framework named SCondi utilizing the messaging service which supports MQTT-an OASIS standard IoT messaging protocol. SCondi provides the notion of context channel as a core feature to support efficient and reliable mechanism for distributing huge context information in the IoT environment. The context channel provides a pluggable filter mechanism that supports effective extraction, tailoring, authentication, and security of information.

S.-H. Lu and Y.-W. Chan proposed a method of combining a peer-to-peer (P2P) overlay network and WSN to develop a bus arrival time prediction system. A P2P overlay network is added to traditional prediction systems to allow for real-time data. Each bus is installed with a sensor, and each bus stop can receive data sent from sensors. Due to the distance limitation of sensors, the sensors on buses and the data-receiving device of a bus station form a single WSN environment. All bus stations and station termini are connected to form a P2P overlay network, which is used to transmit real-time bus information and predict bus arrival times. Through the WSN technology, bus stations retrieve data from buses and transmit these data to subsequent bus stations to estimate the bus arrival times. This approach can be a powerful tool for monitoring and predicting traffic conditions.

S. Kang et al. proposed a group key sharing scheme and efficient rekeying methods for frequent membership changes from network dynamics. The proposed method enables the group members to simply establish a group key and provide high flexibility for dynamic group changes such as member joining or leaving and group merging or partition. They conduct mathematical evaluation with other group key management protocols and finally prove its security by demonstrating group key secrecy, backward and forward secrecy, key independence, and implicit key authentication under the decisional Diffie-Hellman (DDH) assumption.

M. K. Song and M. K. Sarker proposed automatic detection of car LPs via image processing techniques based on classifier or machine learning algorithms. In this paper, they propose a real-time and robust method for LPD systems using the two-stage adaptive boosting (AdaBoost) algorithm combined with different image preprocessing techniques.
Haar-like features are used to compute and select features from LP images. The AdaBoost algorithm is used to classify parts of an image within a search window by a trained strong classifier as either LP or non-LP. Adaptive thresholding is used for the image preprocessing method applied to those images that are of insufficient quality for LPD. This method is of a faster speed and higher accuracy than most of the existing methods used in LPD. Experimental results demonstrate that the average LPD rate is $98.38 \%$ and the computational time is approximately $49 \mathrm{~ms}$.

B. Lee and N. Park, International Association of Lighthouse Authorities (IALA), are developing the standard intersystem VTS exchange format (IVEF) protocol for exchange of navigation and vessel information between VTS systems and between VTS and vessels. VTS (vessel traffic system) is an important marine traffic monitoring system which is designed to improve the safety and efficiency of navigation and the protection of the marine environment. And the demand of inter-VTS networking has been increased for realization of e-navigation as shore side collaboration for maritime safety. And IVEF (inter-VTS data exchange format) for inter-VTS network has become a hot research topic of VTS system. Currently, the IVEF developed by the International Association of Lighthouse Authorities (IALA) does not include any highly trusted certification technology for the connectors. The output of standardization is distributed as the IALA recommendation $\mathrm{V}-145$, and the protocol is implemented with an open source. The IVEF open source, however, is the code used to check the functions of standard protocols. It is too slow to be used in the field and requires a large memory. And the vessel traffic information requires high security since it is highly protected by the countries. Therefore, this paper suggested the authentication protocol to increase the security of the VTS systems using the main certification server and IVEF.

B. Wang et al. developed a novel model and protocol used in some specific scenarios, in which the participants of multiple groups with different permissions can finish the signature together. They applied the secret sharing scheme based on difference equation to the private key distribution phase and secret reconstruction phrase of their threshold signature scheme. In addition, their scheme can achieve the signature success because of the punishment strategy of the repeated rational secret sharing. Besides, the bit commitment and verification method used to detect players' cheating behavior acts as a contributing factor to prevent the internal fraud.

D. Seo et al. presented the Korean spine database and automatic surface mesh intersection algorithm to construct e-spine. To date, the Korean spine database has collected spine data from 77 cadavers and 298 patients. The spine data consists of 2D images from CT, MRI, or X-ray, 3D shapes, geometry data, and property data. The volume and quality of the Korean spine database are now the world's highest ones. In addition, their triangular surface mesh intersection algorithm automatically remeshes the spine-implant intersection model to make it valid for finite element analysis (FEA). This makes it possible to run the FEA using the spine-implant mesh model without any manual effort. 
L.-P. Hung et al. proposed a new framework using radio frequency identification (RFID) technology for a mobilized surgical process monitoring system. Through the active tag, an application management system used before, during, and after the surgical processes has been proposed. The concept of signal level matrix, SLM, was proposed to accurately identify patients and dynamically track patients' location. By updating patient's information real time, the preprocessing time needed for various tasks and incomplete transfers among departments can be reduced, the medical resources can be effectively used, unnecessary medical disputes can be reduced, and more comprehensive health care environment can be provided. The feasibility and effectiveness of their proposed system are demonstrated with a number of experimental results.

K. Hwang and S.-H. Yoon provided a well-defined taxonomy of low-power listening protocols by examining in detail the existing low-power sensor network protocols and evaluation results. It will also be very useful for helping M2M designers understand specific features of low-power media access control protocols as they design new M2M networks.

D. Jung et al. proposed the workflow scheduling scheme that reduces the out-of-bid situation. Consequently, the total task completion time is decreased. The simulation results reveal that, compared to various instance types, their scheme achieves performance improvements in terms of an average combined metric of $12.76 \%$ over workflow scheme without considering the processing rate. However, the cost in their scheme is higher than an instance with low performance and is lower than an instance with high performance.

H.-J. Jeong et al. propose a multilayered quad rotor control method that can move the quad rotor to the desired goal while resisting disturbance. The proposed control system is modular, convenient to design and verify, and easy to extend. It comprises three layers: a physical layer, a displacement control layer, and an attitude control layer. The displacement control layer considers the movement of the vehicle, while the attitude control layer controls its attitude. The physical layer deals with the physical operation of the vehicle. The two control layers use a mathematical method to provide minute step-by-step control. The proposed control system effectively combines the three layers to achieve drift stabilization.

K.-S. Wong and M. H. Kim studied a self-awareness data collection protocol to raise the confidence of the respondents when submitting their personal data to the data collector. Their self-awareness protocol requires each respondent to help others in preserving individual privacy. The communication (respondents and data collector) and collaboration (among respondents) in their solution will be performed automatically.

H. K. Lee et al. provided the following contributions; they presented a service design for an adaptive STB that decreases the dependence among scalable layers. Their adaptive STB converts the receiving scalable streams with high dependency into scalable streams with low dependency. As a result, it decreases the indirect loss of media data and increases streaming service performance even over mobile networks. They then analyzed a media scheme to convert scalable streams.
S. Sivamani et al. proposed a smart logistics service model for providing user-centric intelligent logistics service by utilizing smartphones in a smart environment. They also develop an OWL based ontology model for the smart logistics for the better understanding among the context information.

S.-H. Chae et al. researched how to reconstruct segmentation region in a small region in order to improve the segmentation results. They generated predicted segmentation of slices using volume data with linear equation and proposed improvement method for small regions using the predicted segmentation. In order to verify the performance of the proposed method, lung region by chest CT images was segmented. As a result of experiments, volume data segmentation accuracy rose from 0.978 to 0.981 and from 0.281 to 0.187 with a standard deviation improvement confirmed.

J.-H. Park et al. provided a higher level of abstraction by integration of the two types of access interface, REST API and MapReduce. The motivation of this research stems from the slower response time for accessing simple RDBMS on Hadoop than direct access to RDMBS. This is because there is overhead to job scheduling, initiating, starting, tracking, and management during MapReduce-based parallel execution. Therefore, they provided a good performance for REST Open API service and for MapReduce, respectively.

D. Jung et al. proposed an estimated interval-based checkpointing (EIC) using weighted moving average. Their scheme sets the thresholds of price and execution time based on history. Whenever the actual price and the execution time cross over the thresholds, the system saves the state of spot instances. The Bollinger Bands is adopted to inform the ranges of estimated cost and execution time for user's discretion. The simulation results reveal that, compared to the HBC and REC, the EIC reduces the number of checkpoints and the rollback time. Consequently, the task execution time has been decreased with EIC by HBC and REC. The EIC also provided the benefit of the cost reduction by $\mathrm{HBC}$ and REC, on average.

D. Kang et al. discussed their approach and confirm the validity of their proposed system for preventing network and application protocol attacks in SCADA senor networks.

J. Song et al. presented a novel approach called TSMC (three-stage memory copy) for live virtual machine migration. In TSMC, memory pages only need to be transmitted twice at most and page fault just occurred in small part of dirty pages. They implement it in Xen and compare it with Xen's original precopy approach. The experimental results under various memory workloads show that TSMC approach can significantly reduce the cumulative migration time and total pages transferred and achieve better network IO performance at the same time.

S.-Y. Ihm et al. propose a new skyline method (called Grid-PPPS) for efficiently handling top- $k$ queries in IoT applications. The proposed method first performs Grid-based partitioning on data space and then partitions it once again using hyperplane projection. Experimental results show that their method improves the index building time compared to the existing state-of-the-art methods.

K. Hwang and S. Nam introduced a DSME beacon scheduling model and present a concrete design model. 
Furthermore, validity and performance of DSME are evaluated through experiments. Based on experiment results, they analyzed the problems and limitations of DSME, presented solutions step by step, and finally proposed an enhanced DSME beacon scheduling model. Through additional experiments, they proved the performance superiority of enhanced DSME.

R. Su et al. compared the PMPGA (parallel multipopulation genetic algorithm) with the multiobjective fuzzy optimization algorithm and differential evolution based algorithm and showed that PMPGA has achieved better result. The method can be widely applied to related high-speed train.

K.-C. Lin et al. proposed a classified model in which an artificial fish swarm algorithm and a support vector machine are combined. ALAN environment with several computers which has been infected by the botnet virus was simulated for testing this model; the packet data of network flow was also collected. The proposed method was used to identify the critical features that determine the pattern of botnet. The experimental results indicated that the method can be used for identifying the essential botnet features and that the performance of the proposed method was superior to that of genetic algorithms.

K.-C. Chu and L.-P. Hung proposed the TRPN model and provided a practical, effective, and adaptive method for risk evaluation. In particular, the defined GRPN function offers a new method to prioritize failure modes in failure mode and effect analysis (FMEA). The different risk preferences considered in the healthcare example show that the modified FMEA model can take into account the various risk factors and prioritize failure modes more accurately.

S.-H. Park et al. proposed energy-efficient probabilistic routing (EEPR) algorithm, which controls the transmission of the routing request packets stochastically in order to increase the network lifetime and decrease the packet loss under the flooding algorithm. The proposed EEPR algorithm adopts energy-efficient probabilistic control by simultaneously using the residual energy of each node and ETX metric in the context of the typical AODV protocol. In the simulations, they verified that the proposed algorithm has longer network lifetime and consumes the residual energy of each node more evenly when compared with the typical AODV protocol.

To overcome this limitation, $\mathrm{H}$. Cho et al. tested the usability of a new conceptual autonomous emergency braking (AEB) system that employs vehicle-to-vehicle (V2V) communication technology in the existing AEB system. To this end, a radar sensor and a driving and communication environment constituting the AEB system were simulated; the simulation was then linked by applying vehicle dynamics and control logic. The simulation results show that the collision avoidance relaxation rate of $\mathrm{V} 2 \mathrm{~V}$ communication-based AEB system was reduced compared with that of existing vehicle-mounted-sensor-based system. Thus, a method that can lower the collision risk of the existing AEB system, which uses only a sensor cluster installed on the vehicle, is realized.

Y.-S. Jeong et al. proposed an efficient and secure mobileIPS (m-IPS) for businesses utilizing mobile devices in mobile environments for human-centric computing. The m-IPS system incorporates temporal spatial awareness in humancentric computing with various mobile devices and checks users' temporal spatial information, profiles, and role information to provide precise access control. And it also can extend application of m-IPS to the Internet of Things (IoT), which is one of the important advanced technologies for supporting human-centric computing environment completely, for real ubiquitous field with mobile devices.

L. Chen et al. studied the exponential stability of the complex dynamical network described by differentially nonlinear equations which couple with time delay and stochastic impulses. Some sufficient conditions are established to ensure pth moment exponential stable for the stochastic impulsive systems (SIS) with time delay. An example with its numerical simulation is presented to illustrate the validation of main results.

Finally, D. Shin et al. developed watch-type device (smart watch) that patients wear and a server system. The smart watch developed includes a GPS, accelerometer, and illumination sensor and can obtain real-time health information by measuring the position of patients, quantity of exercise, and amount of sunlight. The server system includes the sensor data analysis algorithm and web server used by the doctor and protector to monitor the sensor data acquired from the smart watch. The proposed data analysis algorithm acquires the exercise information and detects the step count in patients' motion acquired from the acceleration sensor and verifies the three cases of fast pace, slow pace, and walking pace, showing $96 \%$ of the experimental results.

\section{Acknowledgments}

Our special thanks go to everybody of Journal of Applied Mathematics. We would like to thank all authors for their contributions to this special issue. We also extend our thanks to the external reviewers for their excellent help in reviewing the manuscripts.

Young-Sik Jeong
Mohammad S. Obaidat
Jianhua Ma
Laurence T. Yang

\section{References}

[1] Y.-S. Jeong, N. Chilamkurti, and L. J. G. Villalba, "Advanced technologies and communication solutions for internet of things," International Journal of Distributed Sensor Networks, vol. 2014, Article ID 896760, 3 pages, 2014.

[2] L. Atzori, A. Iera, and G. Morabito, "The internet of things: a survey," Computer Networks, vol. 54, no. 15, pp. 2787-2805, 2010.

[3] Y.-S. Jeong and J. H. Park, "High availability and efficient energy consumption for cloud computing service with grid infrastructure," Computers and Electrical Engineering, vol. 39, no. 1, pp. 15-23, 2013.

[4] Gartner, Gartner's Hype Cycle Special Report for 2011, Gartner, 2012, http://www.gartner.com/technology/research/hypecycles/. 
[5] H. Ning and Z. Wang, "Future internet of things architecture: like mankind neural system or social organization framework?" IEEE Communications Letters, vol. 15, no. 4, pp. 461-463, 2011. 


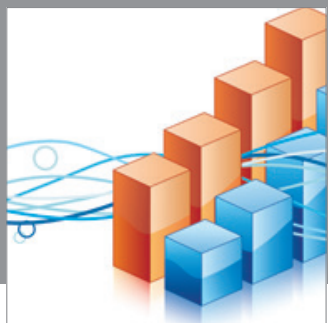

Advances in

Operations Research

mansans

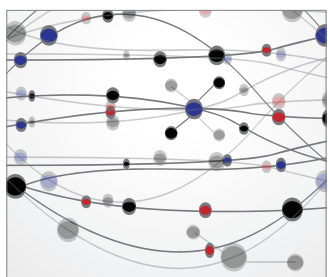

The Scientific World Journal
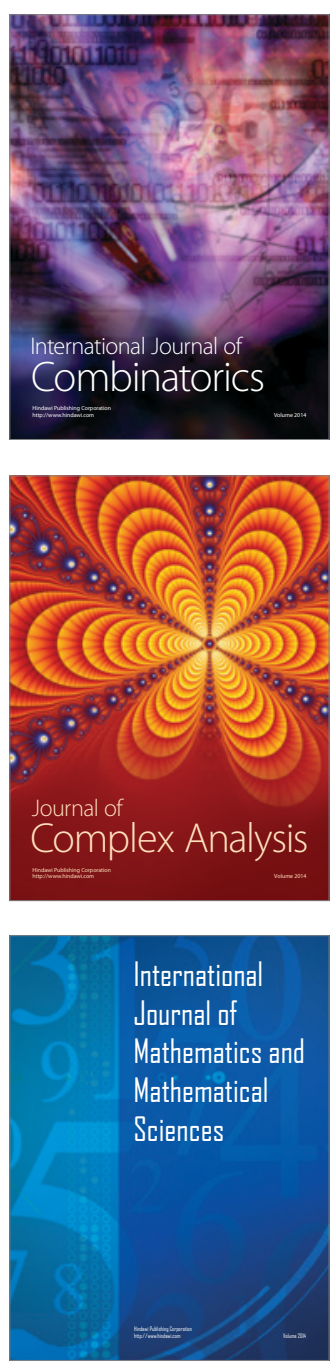
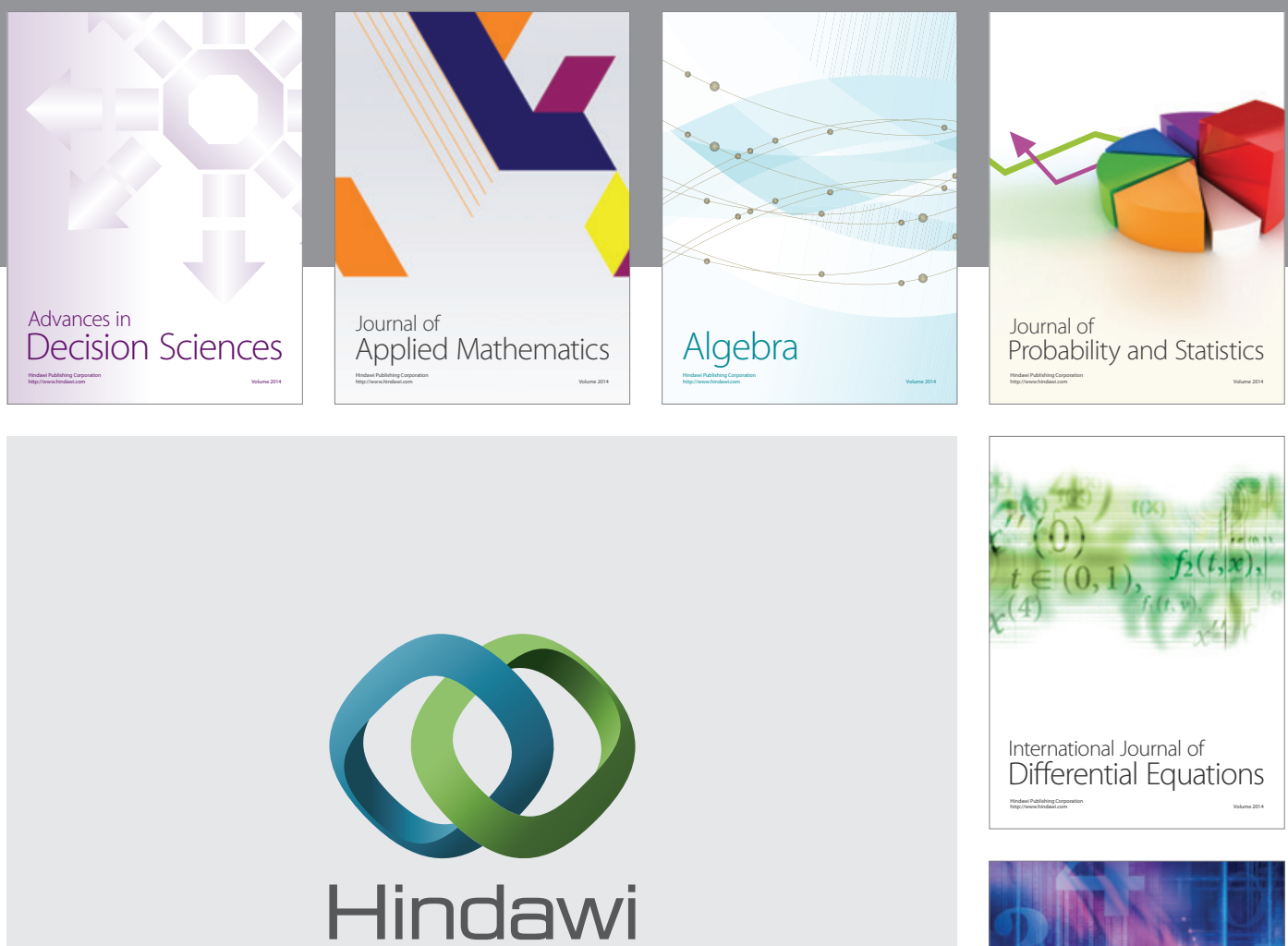

Submit your manuscripts at http://www.hindawi.com
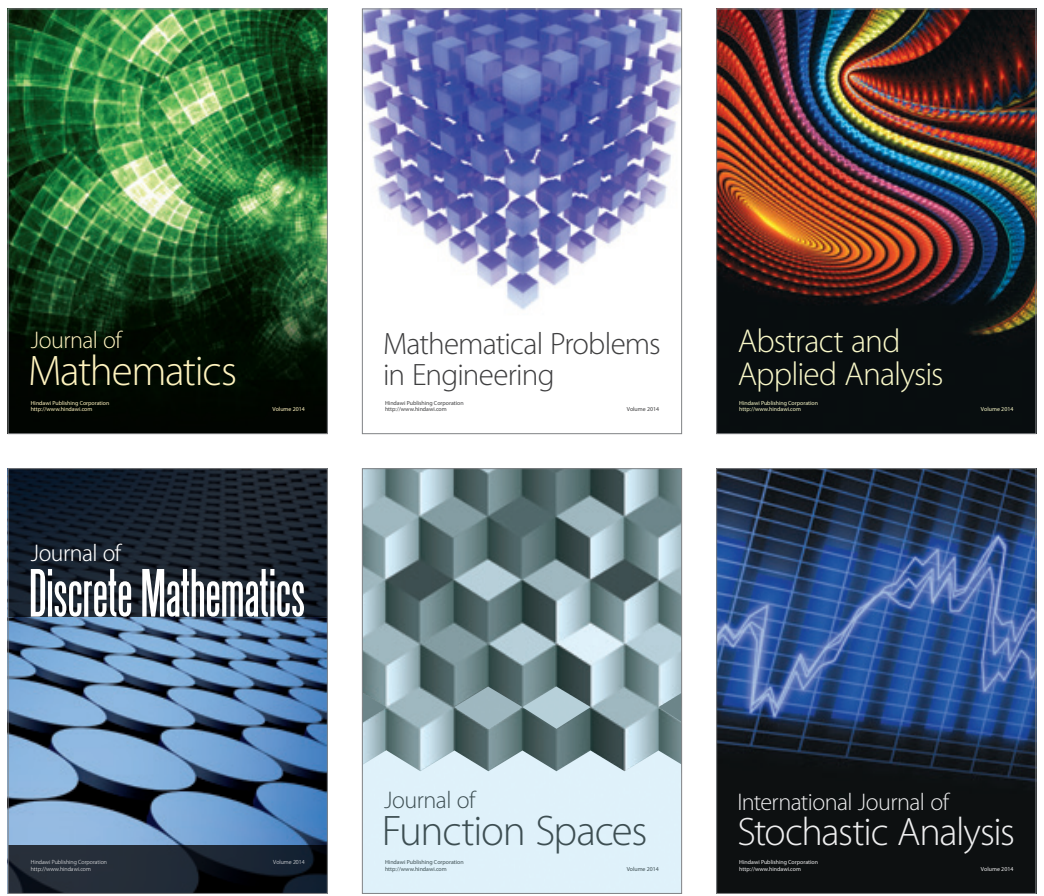

Journal of

Function Spaces

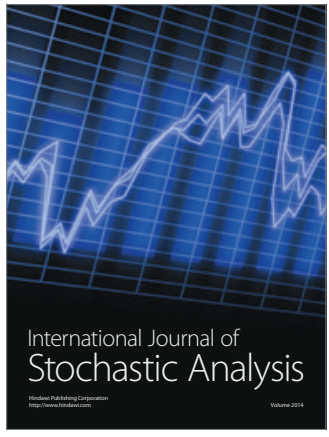

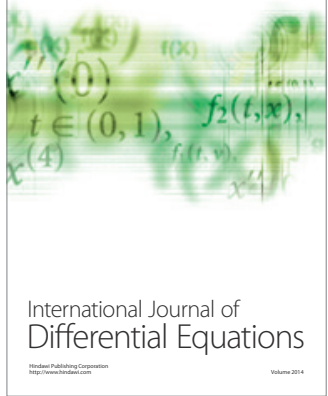
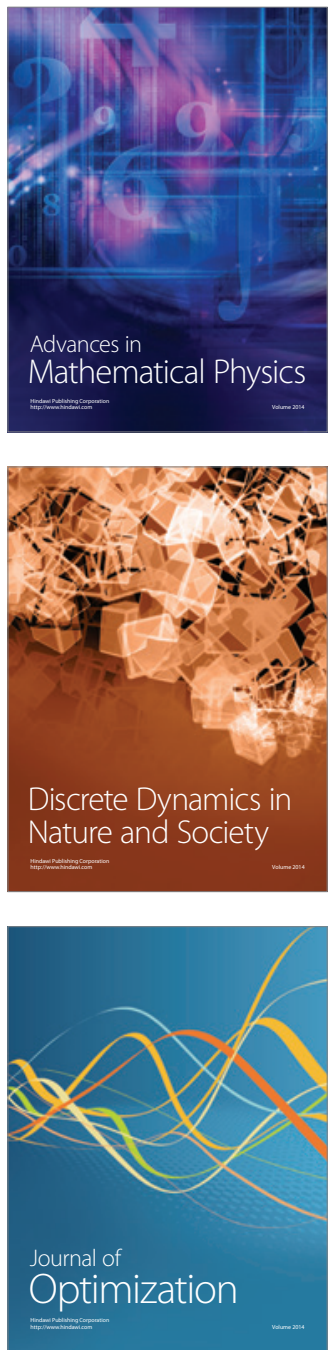